\title{
Development of species diagnostic SNP markers for quality control genotyping in four rice (Oryza L.) species
}

\author{
Marie Noelle Ndjiondjop • Kassa Semagn (D) - Jianwei Zhang • \\ Arnaud Comlan Gouda • Sèdjro Bienvenu Kpeki • Alphonse Goungoulou • \\ Peterson Wambugu • Khady Nani Dramé • Isaac Kofi Bimpong • Dule Zhao
}

Received: 22 June 2018 / Accepted: 17 September 2018 / Published online: 24 October 2018

(C) The Author(s) 2018

\begin{abstract}
Species misclassification (misidentification) and handling errors have been frequently reported in various plant species conserved at diverse gene banks, which could restrict use of germplasm for correct purpose. The objectives of the present study were to (i) determine the extent of genotyping error (reproducibility) on DArTseq-based single-nucleotide polymorphisms (SNPs); (ii) determine the proportion of misclassified accessions across 3134 samples representing three African rice species complex (Oryza glaberrima, $O$. barthii, and $O$. longistaminata) and an Asian rice (O. sativa), which are conserved at the AfricaRice gene bank; and (iii) develop species- and sub-species (ecotype)-specific diagnostic SNP markers for rapid and low-cost quality control (QC) analysis. Genotyping error estimated from 15 accessions, each replicated from 2 to 16 times, varied from 0.2 to $3.1 \%$, with an overall average of $0.8 \%$. Using a total of 3134
\end{abstract}

Electronic supplementary material The online version of this article (https://doi.org/10.1007/s11032-018-0885-z) contains supplementary material, which is available to authorized users.

M. N. Ndjiondjop $(\bowtie) \cdot K$. Semagn $\cdot$ A. C. Gouda • S. B. Kpeki · A. Goungoulou • I. K. Bimpong • D. Zhao M'bé Research Station, Africa Rice Center (AfricaRice), 01 B.P. 2551, Bouaké 01, Côte d'Ivoire

e-mail: m.ndjiondjop@ cgiar.org

K. Semagn $(\bowtie)$

Department of Agricultural, Food and Nutritional Science, University of Alberta, 4-10 Agriculture/Forestry Centre, Edmonton, Alberta T6G 2P5, Canada e-mail: fentaye@ualberta.ca accessions genotyped with 31,739 SNPs, the proportion of misclassified samples was $3.1 \%$ (97 of the 3134 accessions). Excluding the 97 misclassified accessions, we identified a total of 332 diagnostic SNPs that clearly discriminated the three indigenous African species complex from Asian rice (156 SNPs), O. longistaminata accessions from both $O$. barthii and $O$. glaberrima (131 SNPs), and $O$. sativa spp. indica from $O$. sativa spp. japonica (45 SNPs). Using chromosomal position, minor allele frequency, and polymorphic information content as selection criteria, we recommended a subset of 24 to 36 of the 332 diagnostic SNPs for routine QC genotyping, which would be highly useful in determining the genetic identity of each species and correct human errors during routine gene bank operations.

Keywords African rice - Asian rice - Cultivated rice . DArTseq - Genotyping by sequencing - Wild rice

\footnotetext{
J. Zhang

Arizona Genomics Institute and The School of Plant Sciences, University of Arizona, Thomas W. Keating Bioresearch Bldg., 1657 E. Helen Street, Tucson, AZ 85721, USA

P. Wambugu

Kenya Agricultural and Livestock Research Organization

(KALRO), Genetic Resources Research Institute, Nairobi, Kenya

K. N. Dramé

AfricaRice Headquarters, 01 BP 4029, Abidjan 01, Côte d'Ivoire
} 


\section{Introduction}

The Consultative Group on International Agricultural Research (CGIAR) gene banks safeguard some of the most widely used collections of crops and trees in the world, which is critical for attaining global development goals to end hunger and improve food and nutrition security. The CGIAR centers have a gene bank platform that enables them to conserve and make available germplasm under the International Plant Treaty and distribute to the global community (http://www.cgiar.org/aboutus/our-programs/cgiar-genebank-platform-2017-2022). Between 2012 and 2016, CGIAR gene banks distributed over half a million accessions for research and breeding purposes across the world. The Africa Rice Center (AfricaRice) and the International Rice Research Institute (IRRI) are the CGIAR centers, which conserve rice (Oryza L.) germplasm. Africa harbors a huge diversity of both cultivated and wild rice species, representing six of the ten known genome types (Wambugu et al. 2013). AfricaRice conserves nearly 22,000 registered rice samples at its gene bank. The collections represent five African indigenous wild species (Oryza barthii, $O$. longistaminata, O. eichingeri, O. punctata, and $O$. brachyantha) and two cultivated species (O. glaberrima and $O$. sativa) (Ndjiondjop et al. 2017). The cultivated species of $O$. glaberrima along with the wild perennial $O$. longistaminata and wild annual $O$. barthii may be considered as a species complex (Ogbe and Williams 1978).

O. glaberrima accounts for approximately $14 \%$ of the collections at AfricaRice, while all other indigenous wild species are represented by smaller number of samples ranging from 1 to 125 accessions. Recently, our group used the Diversity Arrays Technology-based genotyping by sequencing (DArTseq) platform (Sansaloni et al. 2011) to characterize O. glaberrima collections. We observed that 44 of 2223 O. glaberrima accessions had unusual SNP calls and were considered outliers. Exclusion of the 44 outliers from the dataset resulted in a large decrease (by 77\%) in the number of polymorphic SNPs from 16,532 in the 2223 accessions to 3834 in the remaining 2179 accessions. The 44 outlier accessions may possess rare alleles, which might make them very different from most of the $O$. glaberrima accessions. Outlier accessions might also have resulted from natural allele introgressions into O. glaberrima from another Oryza species (Jones et al. 1997; Semon et al. 2005; Orjuela et al. 2014), especially $O$. barthii, which is presumed to be the wild ancestor of the cultivated O. glaberrima (Ogbe and Williams 1978). In such cases, accessions identified as $O$. glaberrima might be genetically intermediate between $O$. glaberrima and $O$. barthii or other species. Human errors might also occur during (i) plant identification while collecting the accessions in their natural habitats due to inadequate taxonomic expertise and (ii) routine gene bank operations, including germplasm acquisition, conservation, regeneration/multiplication, DNA preparation, and/or genotyping. Proper taxonomic classification and identification of germplasm prior to seed banking is critically important but remains a great challenge for gene bank managers due to heavy dependence on morphological characters that are less accurate in cases where there is limited phenotypic diversity, trait ambiguity, and their variability due to genotype-byenvironment interactions (Ge et al. 2001). Certain Oryza species are closely related, increasing the probability of misidentification, which can easily be avoided using genomic tools for accurate species identification.

Misclassification (misidentification) has been reported in several species, including Oryza glaberrima, $O$. sativa, and $O$. barthii (Orjuela et al. 2014), other wild rice species (Buso et al. 2001), Dioscorea spp. (Girma et al. 2012), and Brassica spp. (Mason et al. 2015). Such types of errors restrict effective use of germplasm for correct purpose in various ways. The latter includes difficulty to tell whether the "true-to-type" accession/ variety has been used for line development, development of mapping populations, molecular breeding, and other genetic studies (Semagn et al. 2012). Quality control (QC) genotyping methods using low-cost, high-throughput, and user-friendly molecular markers have been developed and implemented in some species for genetic purity and genetic identity/authentication (Semagn et al. 2012; Cullingham et al. 2013; Frey et al. 2013; Curk et al. 2015; Ertiro et al. 2015; Chen et al. 2016). Species discriminating markers have also been reported in few plant species (Balasaravanan et al. 2006; Cullingham et al. 2013; Curk et al. 2015) and are finding great application in gene banks where numerous cases of misidentification have been reported (Mason et al. 2015). In rice, Kshirsagar et al. (2014) recommended 12 inter-simple sequence repeats (ISSRs) to serve as varietal diagnostic markers to assess the genetic variability of $48 O$. sativa genotypes. Joshi et al. (2000) screened 30 ISSR markers for their polymorphism on 42 genotypes representing 17 wild Oryza species, $O$. glaberrima and O. sativa, and reported few species- 
specific ISSRs. Chen et al. (2017) genotyped a total of 53 samples, including $O$. glaberrima (18) and $O$. sativa (23), with 33 simple sequence repeat (SSR) markers and reported 10 SSRs that displayed different allelic profiles between the two species. Zhao et al. (2009) genotyped 103 O. rufipogon accessions, 10 O. sativa spp. indica, and $10 O$. sativa spp. japonica cultivars with 123 intron length polymorphism (ILP) markers of which 57 of the markers were found to be highly subspecies-specific between $O$. sativa spp. indica and $O$. sativa spp. japonica. Chin et al. (2007) screened a total of 765 sequence tag sites (STS) using genomic DNA of 15 O. sativa spp. indica and $15 O$. sativa spp. japonica varieties and identified 67 markers for their subspecies specificity. However, species- and subspecies-specific markers reported in previous studies are of limited value to serve as diagnostic markers for several reasons: (i) they were recommended based on very small sample size, (ii) all authors used agarose gels for fragment separation that not only has poor resolution in discriminating genotypes that differ by small allele sizes but also the method is tedious and very low throughput; and (iii) some of the markers are dominant and do not discriminate heterozygous and homozygous loci.

The availability of next-generation sequencing-based genotyping technologies, such as genotyping by sequencing (GBS) (Elshire et al. 2011) and the diversity arrays technology-based sequencing (DArTseq) platform (Sansaloni et al. 2011), have made single-nucleotide polymorphisms (SNPs) very popular for various applications. Some of the advantages of SNPs include low assay cost, high genomic abundance, bi-allelic inheritance, potential for high-throughput analysis, and relatively low genotyping error rates (Rafalski 2002; Schlotterer 2004; McCouch et al. 2012). IRRI recommended a panel of ten SNPs for low-cost QC genotyping for parent-offspring (hybridity) testing and line verification in $O$. sativa spp. indica genotypes (http://gsl.irri.org/genotyping/qualitycontrol-panel/indica-rice-qc-10-snp-panel). It also suggested a panel of 24 SNPs for global QC genotyping in rice. However, the detailed methodology used in selecting the two SNP panels for QC genotyping and their relevance to serve as species and subspecies (ecotype) discriminatory marker set are not currently available. Therefore, the objectives of the present study were to (a) determine the extent of genotyping error (reproducibility) in DArTseq and the proportion of misclassified accessions across four rice species $(O$. glaberrima, $O$. sativa, $O$. barthii, and $O$. longistaminata) and (b) develop a set of species- and subspecies (ecotype)-specific diagnostic SNP markers for low-cost QC genotyping to minimize errors during routine gene bank operations.

\section{Materials and methods}

The two initial sets of germplasm used in the present study are summarized in Supplementary Table S1. The first set consisted of 117 template control DNA samples from 15 accessions, with each accession represented between two to 16 DNA samples. The second set consisted of 3134 samples from 3097 accessions, which represent $O$. longistaminata (20), O. barthii (51), $O$. glaberrima (2422), and O. sativa (558) and genotypes derived from interspecific crosses between $O$. glaberrima and $O$. sativa (83). O. longistaminata was represented by smaller sample size due to limitation on the number of available collections at the AfricaRice gene bank. Thirty-seven accessions were used twice from original and regenerated seed lots to assess the level of human errors during routine gene bank operations. The interspecific genotypes, commonly referred as New Rice for Africa (NERICA), genetically resemble their recurrent $O$. sativa parents. Genomic DNA was extracted from a single plant per sample from 3-weekold seedlings grown in a screenhouse. The detailed methodology used for DNA extraction, SNP genotyping using DArTseq ${ }^{\mathrm{TM}}$, and imputation were described previously (Ndjiondjop et al. 2017). The 117 template DNA samples were randomly placed across 35 of 96well plates used for genotyping the entire germplasm in this study. For each accession, we received 31,739 imputed SNPs from DArT Pty Ltd., Australia (http://www. diversityarrays.com), of which $82.3 \%$ of the markers (26,133 SNPs) were mapped to the 12 rice chromosomes, while the remaining $17.7 \%$ were not assigned into any of the chromosomes.

All statistical analyses were performed after filtering the SNP data of the two sets of germplasm using a minor allele frequency (MAF) of 0.01 in TASSEL v.5.2.43 software (Bradbury et al. 2007). An identity-by-state (IBS)-based genetic distance matrices were computed between pair of DNA samples of each data set using TASSEL v.5.2.43. The genetic distance matrix in the first data set was used as an indicator of genotyping error (reproducibility), whereby pairs of DNA samples from the same accession are expected to have a zero distance 
(no mismatch); values exceeding zero are indicative of genotyping errors with larger values showing higher proportion of mismatch between template DNA samples of the same accession. In the second dataset, the genetic distance matrix was used for cluster analysis to understand the extent of species misclassification due to human error during plant identification while collecting the accessions in the field, germplasm acquisition, and/or routine gene bank activities. Cluster analysis was performed using the neighbor-joining method implemented in molecular evolutionary genetics analysis (MEGA) v.7.0 (Kumar et al. 2016). We also used principal component analysis (PCA) implemented in TASSEL v.5.2.43. The first two principal components from the PCA were plotted for visual examination in XLSTAT 2012 (Addinsof, New York, USA; www.xlstat.com) using the scatter plot option and species/ecotype as a categorical variable. Accessions belonging to the same species/ecotype tend to cluster more closely together than those from other species/ecotype irrespective of the algorithms used for computing genetic distance matrices and the multivariate method used for analyzing genetic relationships.

For low-cost and routine quality control genotyping, diagnostic SNPs were identified from a third dataset created after excluding all misclassified accessions in the second dataset by comparing SNPs that had the same allele across all accessions of the same species/ecotype/ ecology against all accessions from a second species/ ecotype/ecology using an algorithm developed by the Arizona Genomics Institute and The School of Plant Sciences, University of Arizona. The selected diagnostic SNPs were then compiled into a fourth dataset for rapid navigation and comparisons between species/ecotype/ ecologies using TASSEL v.5.2.43 and Flapjack v1.16.10.31 (Milne et al. 2010).

\section{Results}

Genotyping error and accession misclassification

After filtering the SNPs using a minor allele frequency of $1 \%$, nearly $74 \%$ of the markers $(23,490$ of 31,739 SNPs $)$ in the first dataset were polymorphic across the 117 template control DNA samples. Genotyping errors across the multiple DNA samples of each of the 15 accessions varied from 0.2 to $3.1 \%$, with an overall average of $0.8 \%$. Genotyping error between pairs of DNA samples of the same accession exceeded $1 \%$ only in three
(WAB0002367, WAB0013445, and WAB0000026) of the 15 accessions (Fig. 1). In the second dataset, 87\% of the markers $(27,645$ of 31,739 SNPs) were polymorphic across the 3134 accessions, each SNP with a minor allele frequency ranging from 0.01 to 0.499 (data not shown). Ninety-seven of the 3134 accessions were misclassified (Supplementary Table S1), which accounts for $3.1 \%$ of the total germplasm evaluated in this study. The 97 misclassified accessions included 64 O. glaberrima, 22 $O$. sativa, 7 O. barthii, and $4 O$. longistaminata. As shown in Supplementary Fig. S1 and Figs. 2 and 3, the misclassified $O$. barthii accessions clustered together with $O$. longistaminata (1), O. glaberrima (2), and $O$. sativa (4), whereas those misclassified accessions from $O$. longistaminata were similar either to O. glaberrima (1) or $O$. barthii (3). The 64 misclassified $O$. glaberrima accessions were clustered with the lowland $O$. sativa (44) and upland $O$. sativa (22) accessions (Fig. 2), while all 22 misclassified $O$. sativa accessions were clustered together with $O$. glaberrima (Fig. 3). Of the 97 misclassified samples, 37 samples were regenerated seed lots, which included O. glaberrima (32), $O$. longistaminata (1), and $O$. sativa (4).

\section{Diagnostic marker identification}

To develop a smaller set of species- or group-specific markers for low-cost and routine QC genotyping, we searched for diagnostic SNPs across 3037 of the 3134 accessions after excluding the 97 misclassified samples. Supplementary Table S2 summarizes the 35 pairs of comparisons that involved the four species, three groups of $O$. sativa (O. sativa spp. indica and japonica and interspecifics) and two ecologies (lowland and upland). The number of diagnostic SNPs identified between pairs of species or groups varied from none to 5640 . We first searched for diagnostic SNPs between indigenous African species complex (O. glaberrima, $O$. barthii, and $O$. longistaminata; $N=2418$ ) as one group and the Asian rice (O. sativa, $N=$ $619)$ as the second group and identified 156 diagnostic SNPs (Supplementary Table S3) that clearly revealed contrasting haplotypes between the two groups. One hundred and thirty-six of the 156 diagnostic SNPs were mapped to the 12 rice chromosomes, while the remaining 20 SNPs were not assigned to any chromosome. The number of mapped diagnostic SNPs between the African species complex and the Asian rice varied from 1 on chromosome 8 to 29 on chromosome 2 . In the second step, we searched for diagnostic SNPs that discriminated between pairs of 


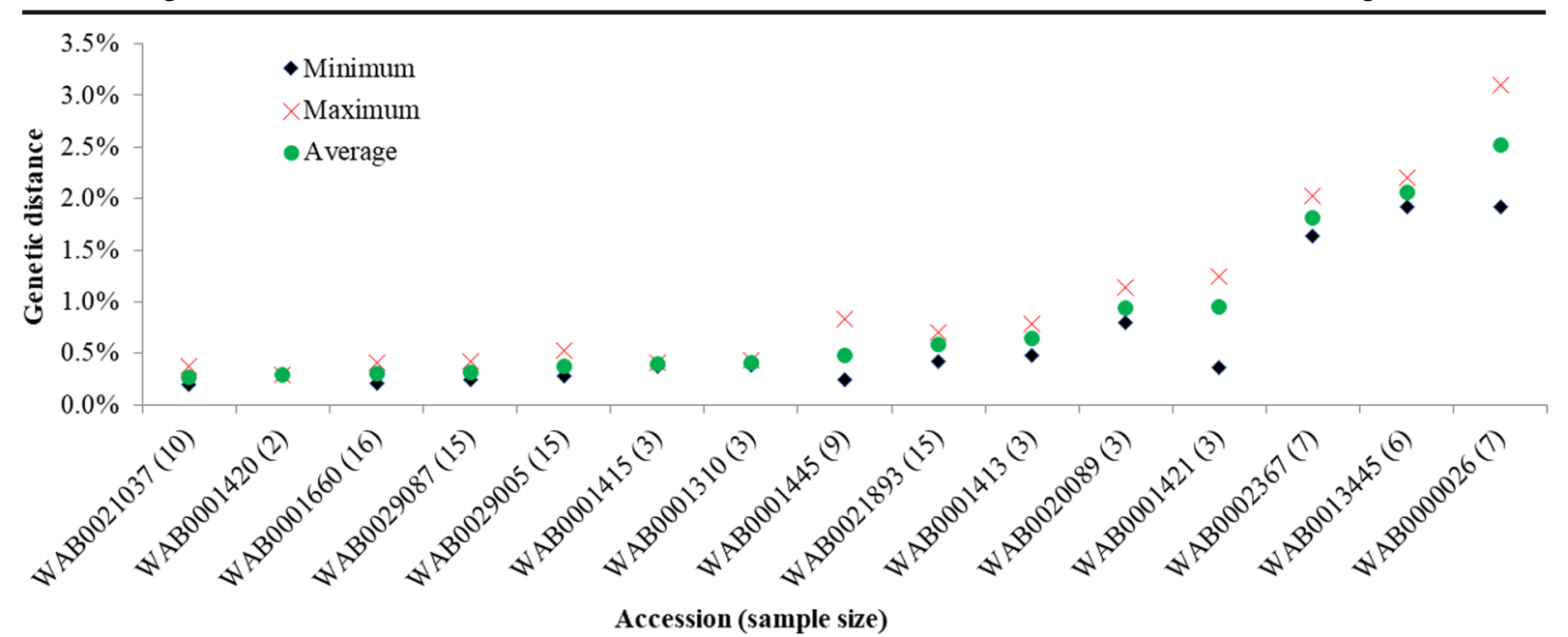

Fig. 1 Comparison of identity-by-state-based genetic distance among pairs of DNA samples from the same accession as a measure of genotyping error of 117 DNA samples from 15 accessions. Each accession was represented from 2 to 16 DNA samples

the three African species complex and between $O$. sativa groups (O. sativa spp. indica and japonica or lowland and upland ecologies). We found that all $O$. longistaminata accessions differed from $O$. barthii and $O$. glaberrima at 649 and 141 SNPs, respectively, of which 131 SNPs were common in both comparisons (Supplementary Table S2, Supplementary Table S2). No diagnostic SNP was identified between $O$. barthii and $O$. glaberrima. Since no marker was found to be diagnostic between $O$. glaberrima and $O$. barthii, the 131 SNPs should be sufficient to serve as diagnostic markers between $O$. longistaminata and $O$. glaberrima/O. barthii accessions. One hundred and ten of the 131 were mapped across the 12 rice chromosomes, with each chromosome consisting of 4 to 29 diagnostic SNPs. Sets of 30 and 45 SNPs discriminating $O$. sativa accessions to lowland or upland ecologies and O. sativa spp. indica or japonica, respectively, were identified (Supplementary Table S2, Supplementary Table S3). The 30 diagnostic SNPs were common across the two ecologies and the two ecotypes, whereas 15 SNPs were diagnostic only between $O$. sativa spp. indica and japonica. No diagnostic SNP was found between NERICA and $O$. sativa spp. indica and japonica, which is expected due to the high genetic similarity of NERICAs with their recurrent $O$. sativa parents with a clear population structure corresponding either to the lowland or upland ecology (Supplementary Fig. S1 and Fig. 2).

Overall, the total number of diagnostic markers identified across the three sets of germplasm was 332 SNPs, and genotyped with 31,7369 SNPs of which 23,490 SNPs were polymorphic. Only three accessions showed genotyping error between pairs of DNA samples greater than $1 \%$

each with MAF and polymorphism information content (PIC) varying from 0.005 to 0.223 and from 0.004 to 0.487 , respectively. Supplementary Fig. 2 shows the chromosomal distribution of 285 of the 332 diagnostic SNPs that were mapped across the 12 rice chromosomes. Based on chromosomal positions and a minimum MAF of 0.175 and a PIC value of 0.150 , we recommend 36 of the 285 diagnostic SNPs mapped across the 12 rice chromosomes for low-cost quality control genotyping (Table 1, Supplementary Table S3). The genotype data of the 36 diagnostic SNPs across the 3037 accessions (excluding the 97 outliers) are given in Supplementary Table S4. As shown in Fig. 4 and Supplementary Table S3, we selected a subset of 14, 11, and 11 SNPs for unambiguous haplotype discrimination of the three African species complex from Asian rice, $O$. longistaminata from both $O$. barthii/O. glaberrima, and lowland $O$. sativa spp. indica from upland $O$. sativa spp. japonica, respectively. To minimize genotyping cost per sample for uniplex assays, however, a smaller subset of even eight diagnostic SNPs per group of germplasm are sufficient for routine QC genotyping, which is discussed in detail in the next section.

\section{Discussion}

Gene bank managers are challenged to ensure the accurate identification of species and maintaining the genetic integrity of collections by preventing human errors 


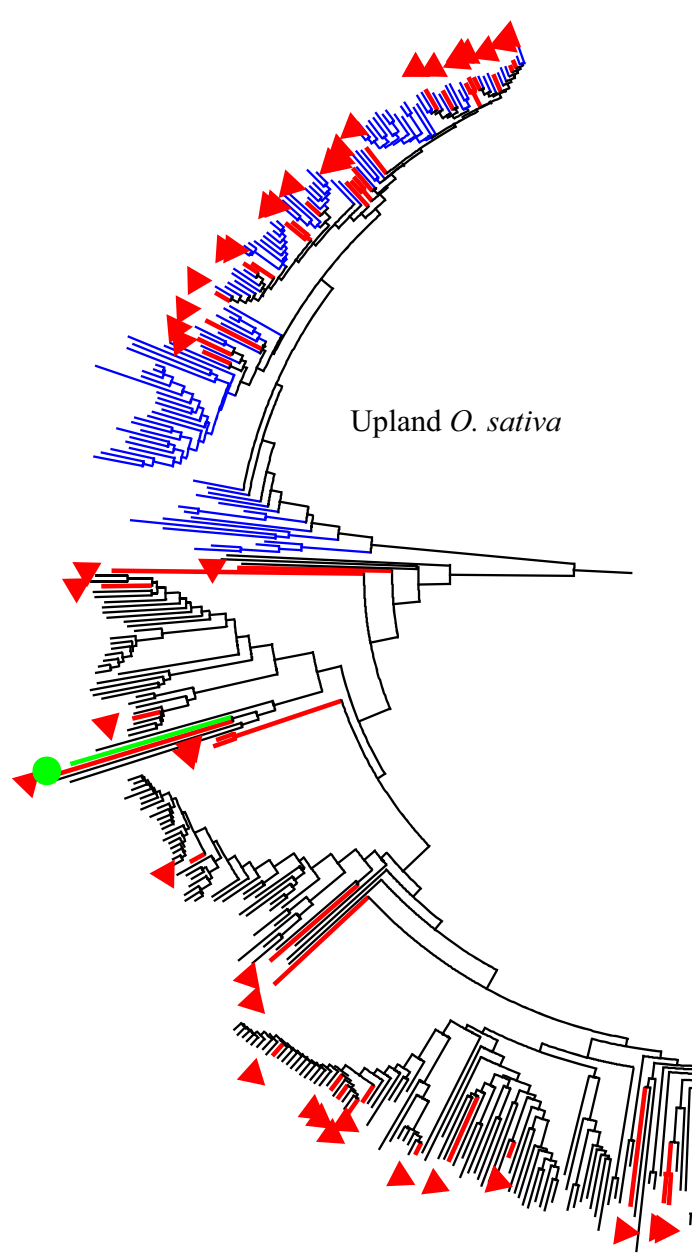

Fig. 2 Neighbor-joining tree of 619 lowland (black) and upland (blue) $O$. sativa accessions based on genetic distance matrix computed from 27,645 polymorphic SNPs to demonstrate 64

during routine gene bank operations, including labeling errors and admixtures during seed handling. Many gene banks do not have the resources to assess every collection for genetic diversity, correct origins, and species classification (Mason et al. 2015). Using a set of 93 to 235 markers, previous studies conducted on rice germplasm conserved at AfricaRice gene bank have reported the presence of admixture between $O$. glaberrima and O. sativa (Semon et al. 2005) and between $O$. glaberrima and $O$. barthii (Orjuela et al. 2014). However, none of those studies have provided evidence of possible human error on such types of admixture. To the best of our knowledge, this is the first extensive study that has explored the extent of human error using misclassified $O$. glaberrima (red) and 4 O. barthii (green) accessions. Thirty-two of the 64 misclassified $O$. glaberrima accessions originated from regenerated seed lots

large set of rice germplasm and identified species- and ecotype-specific diagnostic SNPs for low-cost and highthroughput QC genotyping. Overall, we found that $3.1 \%$ of the 3134 accessions used in the present study were misclassified (Supplementary Table S1). Such misclassification could be due to genotyping errors caused by the DArTseq technology, errors during germplasm collection, and/or during routine gene bank operations. The quality of DArTseq markers is assessed based on call rates and reproducibility scores of template control DNA samples, which are provided by DArT Pty Ltd. (the genotyping service provider). The reproducibility score of DArTseq markers is the proportion of technical replicate assay pairs for which the marker 
Fig. 3 Neighbor-joining tree of O. glaberrima (black) accessions with misclassified $O$. sativa (blue) and $O$. barthii (green) accessions based on genetic distance matrix computed from 27,645 polymorphic SNPs

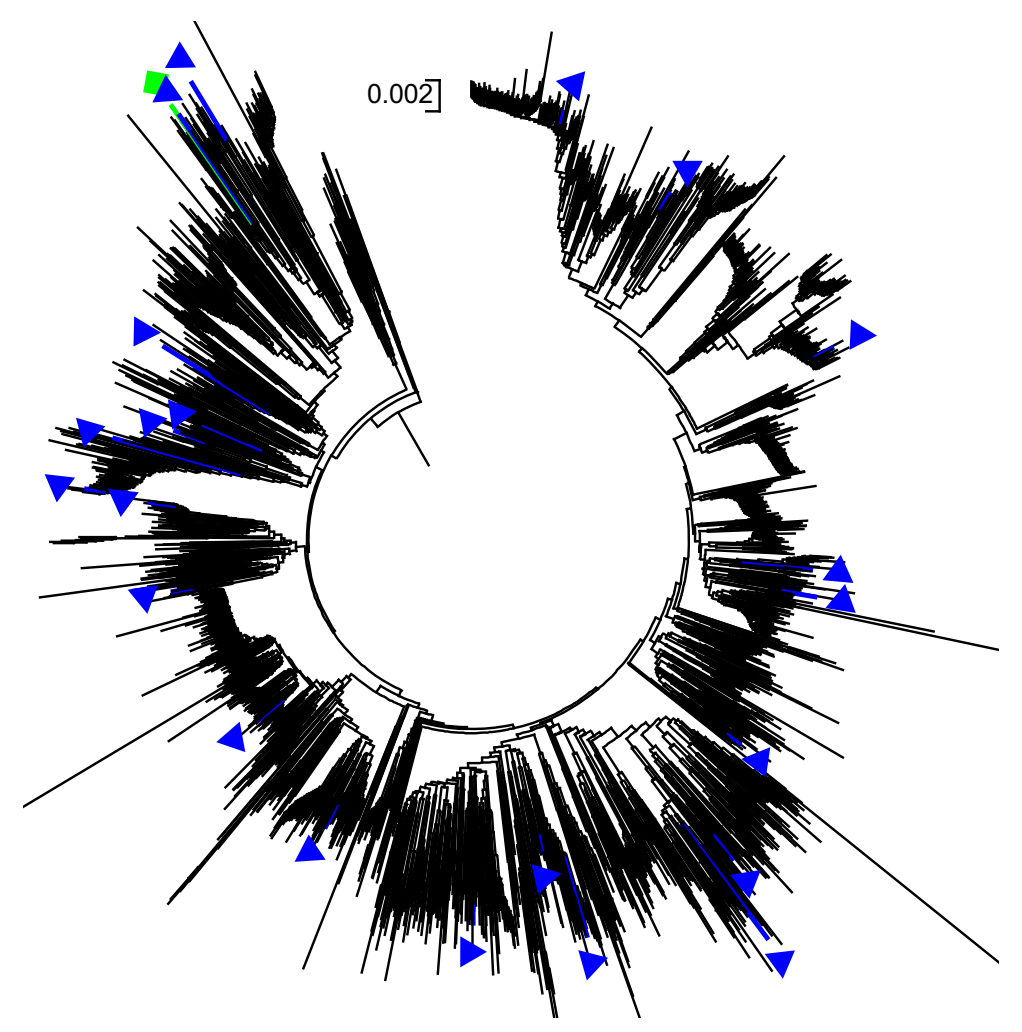

score is consistent, which has been reported to be 99 100\% (Baloch et al. 2017; Melville et al. 2017). In such cases, genotyping error accounts for a maximum of $1 \%$ only. In the current study, the average genotyping error between pairs of template control DNA samples from 15 accessions was $0.8 \%$, which translates into an average reproducibility of $99.2 \%$ (range 96.9-99.8\%). Between pairs of DNA samples from three accessions, however, larger error rates were noted (Fig. 1). Overall, the average genotyping error for DArTseq SNPs in our study was greater than the values reported in other studies using the same platform (Baloch et al. 2017; Melville et al. 2017) but was lower than the 2.0-2.4\% reported using other SNP genotyping platforms (Yan et al. 2010; Semagn et al. 2014) and DNA sequencing (Cubry et al. 2018). Our results together with others suggest a very minimal effect of genotyping error on the proportion of misclassification observed in the present study.

To trace the source of human errors during routine gene bank operations, we compared the genotypic data of the original collections and regenerated seed lot of 37 accessions (Supplementary Table S1). In 35 of the 37 original seed lots, we found that accessions originating from the same species clustered as expected, while two original seed lots were misclassified, probably due to species misclassification during germplasm collection. All the 37 regenerated seed lots were misclassified, which might be caused by labeling errors made during seed regeneration/multiplication and seed processing/ handling. The proportion of misclassification observed in our study was much lower than the 5-21\% misclassification reported in other studies (Buso et al. 2001; Girma et al. 2012; Orjuela et al. 2014; Mason et al. 2015). Orjuela et al. (2014) reported misclassification of 13 of 266 O. glaberrima accessions (4.9\%), which were supposed to be $O$. sativa; however, the proportion of misclassification in their study seems over $20 \%$, as there were several $O$. barthii accessions that were clustered with $O$. glaberrima in both neighbor-joining phylogenetic analysis and PCA. Buso et al. (2001) studied 230 cultivated and wild Oryza species using random amplified polymorphic DNA, flow cytometry, and chromosome counting and reported $8 \%$ of misclassification either taxonomically or as a result of contamination. Mason et al. (2015) genotyped 180 lines from five Brassica species sourced from the Australian Grains Genebank using the Illumina Infinium Brassica 60K SNP array, which included 76 suspected misclassified 
Table 1 Summary of the 36 diagnostic SNPs recommended for routine quality control genotyping in three sets of rice germplasm. (See Supplementary Table S3 for details, including major and minor alleles and sequences)

\begin{tabular}{|c|c|c|c|c|c|}
\hline Clone (SNP) ID & Chromosome* & $\begin{array}{l}\text { Physical } \\
\text { position } \\
(\mathrm{bp})^{*}\end{array}$ & $\begin{array}{l}\text { Minor } \\
\text { allele } \\
\text { frequency }\end{array}$ & $\begin{array}{l}\text { Polymorphism } \\
\text { information content } \\
\text { (PIC) }\end{array}$ & Comment** \\
\hline 19323604|F|0-33:G>A-33:G>A & 1 & $13,006,094$ & 0.175 & 0.179 & $\begin{array}{l}\text { Diagnostic between lowland } \\
\text { O. sativa } \text { spp. indica and upland } \\
\text { japonica }\end{array}$ \\
\hline $3048732|\mathrm{~F}| 0-43: \mathrm{C}>\mathrm{T}-43: \mathrm{C}>\mathrm{T}$ & 2 & $33,878,778$ & 0.175 & 0.155 & $\begin{array}{l}\text { Diagnostic between lowland } \\
\text { O. sativa } \text { spp. indica and upland } \\
\text { japonica }\end{array}$ \\
\hline 5143398|F|0-19:C >A-19:C >A & 3 & 878,615 & 0.176 & 0.248 & $\begin{array}{l}\text { Diagnostic between lowland } \\
\text { O. sativa spp. indica and upland } \\
\text { japonica }\end{array}$ \\
\hline $3061709|\mathrm{~F}| 0-55: \mathrm{T}>\mathrm{C}-55: \mathrm{T}>\mathrm{C}$ & 4 & $27,782,473$ & 0.175 & 0.150 & $\begin{array}{l}\text { Diagnostic between lowland } \\
\text { O. sativa spp. indica and upland } \\
\text { japonica }\end{array}$ \\
\hline $19322100|\mathrm{~F}| 0-34: \mathrm{G}>\mathrm{A}-34: \mathrm{G}>\mathrm{A}$ & 5 & $7,309,276$ & 0.175 & 0.192 & $\begin{array}{l}\text { Diagnostic between lowland } \\
\text { O. sativa spp. indica and upland } \\
\text { japonica }\end{array}$ \\
\hline $5398605|\mathrm{~F}| 0-5: \mathrm{A}>\mathrm{C}-5: \mathrm{A}>\mathrm{C}$ & 6 & $18,012,850$ & 0.176 & 0.177 & $\begin{array}{l}\text { Diagnostic between lowland } \\
\text { O. sativa spp. indica and upland } \\
\text { japonica }\end{array}$ \\
\hline $5408937|\mathrm{~F}| 0-68: \mathrm{G}>\mathrm{A}-68: \mathrm{G}>\mathrm{A}$ & 7 & $7,357,816$ & 0.175 & 0.181 & $\begin{array}{l}\text { Diagnostic between lowland } \\
\text { O. sativa spp. indica and upland } \\
\text { japonica }\end{array}$ \\
\hline $3054571|\mathrm{~F}| 0-22: \mathrm{G}>\mathrm{T}-22: \mathrm{G}>\mathrm{T}$ & 8 & $18,620,171$ & 0.177 & 0.168 & $\begin{array}{l}\text { Diagnostic between lowland } \\
\text { O. sativa spp. indica and upland } \\
\text { japonica }\end{array}$ \\
\hline $3764294|\mathrm{~F}| 0-38: \mathrm{T}>\mathrm{G}-38: \mathrm{T}>\mathrm{G}$ & 9 & $18,312,602$ & 0.176 & 0.159 & $\begin{array}{l}\text { Diagnostic between lowland } \\
\text { O. sativa spp. indica and upland } \\
\text { japonica }\end{array}$ \\
\hline $3061808|\mathrm{~F}| 0-33: \mathrm{G}>\mathrm{A}-33: \mathrm{G}>\mathrm{A}$ & 11 & $11,776,508$ & 0.176 & 0.257 & $\begin{array}{l}\text { Diagnostic between lowland } \\
\text { O. sativa spp. indica and upland } \\
\text { japonica }\end{array}$ \\
\hline $3451491|\mathrm{~F}| 0-7: \mathrm{A}>\mathrm{G}-7: \mathrm{A}>\mathrm{G}$ & 12 & $12,303,324$ & 0.176 & 0.161 & $\begin{array}{l}\text { Diagnostic between lowland } \\
\text { O. sativa spp. indica and upland } \\
\text { japonica }\end{array}$ \\
\hline 3999042|F|0-64:A>T-64:A>T & 1 & $3,338,923$ & 0.225 & 0.343 & $\begin{array}{l}\text { Diagnostic between } \\
\text { O. longistaminata and } \\
\text { O. glaberrima/O. barthii }\end{array}$ \\
\hline $5400312|\mathrm{~F}| 0-7: \mathrm{T}>\mathrm{C}-7: \mathrm{T}>\mathrm{C}$ & 2 & $14,337,444$ & 0.224 & 0.346 & $\begin{array}{l}\text { Diagnostic between } O . \\
\text { longistaminata and } \mathrm{O} . \\
\text { glaberrima/O. barthii }\end{array}$ \\
\hline $3772372|\mathrm{~F}| 0-31: \mathrm{A}>\mathrm{C}-31: \mathrm{A}>\mathrm{C}$ & 3 & $32,703,627$ & 0.222 & 0.341 & $\begin{array}{l}\text { Diagnostic between } \\
\text { O. longistaminata and } \\
\text { O. glaberrima/O. barthii }\end{array}$ \\
\hline $5404734|\mathrm{~F}| 0-26: \mathrm{G}>\mathrm{C}-26: \mathrm{G}>\mathrm{C}$ & 4 & $12,064,222$ & 0.224 & 0.341 & $\begin{array}{l}\text { Diagnostic between } \\
\text { O. longistaminata and } \\
\text { O. glaberrima/O. barthii }\end{array}$ \\
\hline 9759889|F|0-13:C >A-13:C>A & 5 & $23,874,724$ & 0.224 & 0.340 & $\begin{array}{l}\text { Diagnostic between } \\
\text { O. longistaminata and } \\
\text { O. glaberrima/O. barthii }\end{array}$ \\
\hline
\end{tabular}


Table 1 (continued)

\begin{tabular}{|c|c|c|c|c|c|}
\hline Clone (SNP) ID & Chromosome* & $\begin{array}{l}\text { Physical } \\
\text { position } \\
(\mathrm{bp})^{*}\end{array}$ & $\begin{array}{l}\text { Minor } \\
\text { allele } \\
\text { frequency }\end{array}$ & $\begin{array}{l}\text { Polymorphism } \\
\text { information content } \\
\text { (PIC) }\end{array}$ & Comment** \\
\hline $3767922|\mathrm{~F}| 0-20: \mathrm{A}>\mathrm{C}-20: \mathrm{A}>\mathrm{C}$ & 6 & $29,415,564$ & 0.224 & 0.342 & $\begin{array}{l}\text { Diagnostic between } \\
\text { O. longistaminata and } \\
\text { O. glaberrima/O. barthii }\end{array}$ \\
\hline $3756907|\mathrm{~F}| 0-10: \mathrm{G}>\mathrm{A}-10: \mathrm{G}>\mathrm{A}$ & 7 & $22,969,163$ & 0.223 & 0.252 & $\begin{array}{l}\text { Diagnostic between } \\
\text { O. longistaminata and } \\
\text { O. glaberrima/O. barthii }\end{array}$ \\
\hline 4392882|F|0-24:G>A-24:G>A & 9 & $17,267,685$ & 0.225 & 0.470 & $\begin{array}{l}\text { Diagnostic between } \\
\text { O. longistaminata and } \\
\text { O. glaberrima/O. barthii }\end{array}$ \\
\hline 5402462|F|0-8:A>G-8:A>G & 10 & $21,079,827$ & 0.224 & 0.339 & $\begin{array}{l}\text { Diagnostic between } \\
\text { O. longistaminata and } \\
\text { O. glaberrima/O. barthii }\end{array}$ \\
\hline $3766781|\mathrm{~F}| 0-33: \mathrm{G}>\mathrm{C}-33: \mathrm{G}>\mathrm{C}$ & 11 & $19,638,886$ & 0.223 & 0.342 & $\begin{array}{l}\text { Diagnostic between } \\
\text { O. longistaminata and } \\
\text { O. glaberrima/O. barthii }\end{array}$ \\
\hline $9760013|\mathrm{~F}| 0-21: \mathrm{G}>\mathrm{C}-21: \mathrm{G}>\mathrm{C}$ & 12 & $17,069,058$ & 0.225 & 0.487 & $\begin{array}{l}\text { Diagnostic between } \\
\text { O. longistaminata and } \\
\text { O. glaberrima/O. barthii }\end{array}$ \\
\hline 9752577|F|0-33:A>T-33:A>T & 1 & $40,671,128$ & 0.219 & 0.344 & $\begin{array}{c}\text { Diagnostic SNP between Africa rice } \\
\text { species complex and Asian rice }\end{array}$ \\
\hline $3449013|\mathrm{~F}| 0-42: \mathrm{C}>\mathrm{T}-42: \mathrm{C}>\mathrm{T}$ & 2 & $2,687,374$ & 0.219 & 0.382 & $\begin{array}{l}\text { Diagnostic SNP between Africa rice } \\
\text { species complex and Asian rice }\end{array}$ \\
\hline 9756975|F|0-38:A>C-38:A>C & 3 & $21,681,288$ & 0.219 & 0.305 & $\begin{array}{c}\text { Diagnostic SNP between Africa rice } \\
\text { species complex and Asian rice }\end{array}$ \\
\hline $3442388|\mathrm{~F}| 0-60: \mathrm{C}>\mathrm{A}-60: \mathrm{C}>\mathrm{A}$ & 4 & $23,879,007$ & 0.219 & 0.262 & $\begin{array}{c}\text { Diagnostic SNP between Africa rice } \\
\text { species complex and Asian rice }\end{array}$ \\
\hline 9759135|F|0-10:T>C-10:T>C & 5 & $20,316,123$ & 0.219 & 0.318 & $\begin{array}{c}\text { Diagnostic SNP between Africa rice } \\
\text { species complex and Asian rice }\end{array}$ \\
\hline 5410301|F|0-24:C>T-24:C >T & 6 & $5,959,340$ & 0.219 & 0.251 & $\begin{array}{c}\text { Diagnostic SNP between Africa rice } \\
\text { species complex and Asian rice }\end{array}$ \\
\hline 3987858|F|0-29:T>A-29:T>A & 7 & $4,661,018$ & 0.219 & 0.349 & $\begin{array}{c}\text { Diagnostic SNP between Africa rice } \\
\text { species complex and Asian rice }\end{array}$ \\
\hline 6997101|F|0-26:G>A-26:G>A & 8 & $8,968,236$ & 0.219 & 0.384 & $\begin{array}{c}\text { Diagnostic SNP between Africa rice } \\
\text { species complex and Asian rice }\end{array}$ \\
\hline $3052158|\mathrm{~F}| 0-20: \mathrm{G}>\mathrm{A}-20: \mathrm{G}>\mathrm{A}$ & 9 & $1,894,850$ & 0.219 & 0.252 & $\begin{array}{c}\text { Diagnostic SNP between Africa rice } \\
\text { species complex and Asian rice }\end{array}$ \\
\hline $3771354|\mathrm{~F}| 0-14: \mathrm{C}>\mathrm{T}-14: \mathrm{C}>\mathrm{T}$ & 9 & $18,345,726$ & 0.219 & 0.339 & $\begin{array}{c}\text { Diagnostic SNP between Africa rice } \\
\text { species complex and Asian rice }\end{array}$ \\
\hline $5400008|\mathrm{~F}| 0-23: \mathrm{G}>\mathrm{A}-23: \mathrm{G}>\mathrm{A}$ & 9 & $21,792,333$ & 0.219 & 0.357 & $\begin{array}{c}\text { Diagnostic SNP between Africa rice } \\
\text { species complex and Asian rice }\end{array}$ \\
\hline $3055213|\mathrm{~F}| 0-32: \mathrm{A}>\mathrm{G}-32: \mathrm{A}>\mathrm{G}$ & 10 & $10,645,458$ & 0.219 & 0.324 & $\begin{array}{c}\text { Diagnostic SNP between Africa rice } \\
\text { species complex and Asian rice }\end{array}$ \\
\hline 5388786|F|0-44:T>A-44:T>A & 11 & $2,335,078$ & 0.219 & 0.386 & $\begin{array}{l}\text { Diagnostic SNP between Africa rice } \\
\text { species complex and Asian rice }\end{array}$ \\
\hline $3995884|\mathrm{~F}| 0-25: \mathrm{G}>\mathrm{A}-25: \mathrm{G}>\mathrm{A}$ & 12 & $25,433,207$ & 0.219 & 0.332 & $\begin{array}{c}\text { Diagnostic SNP between Africa rice } \\
\text { species complex and Asian rice }\end{array}$ \\
\hline
\end{tabular}

*Chromosome numbers and physical positions in base pairs (bp) are based on Rice_v9

**African rice species complex refers to O. glaberrima, O. barthii, and O. longistaminata 


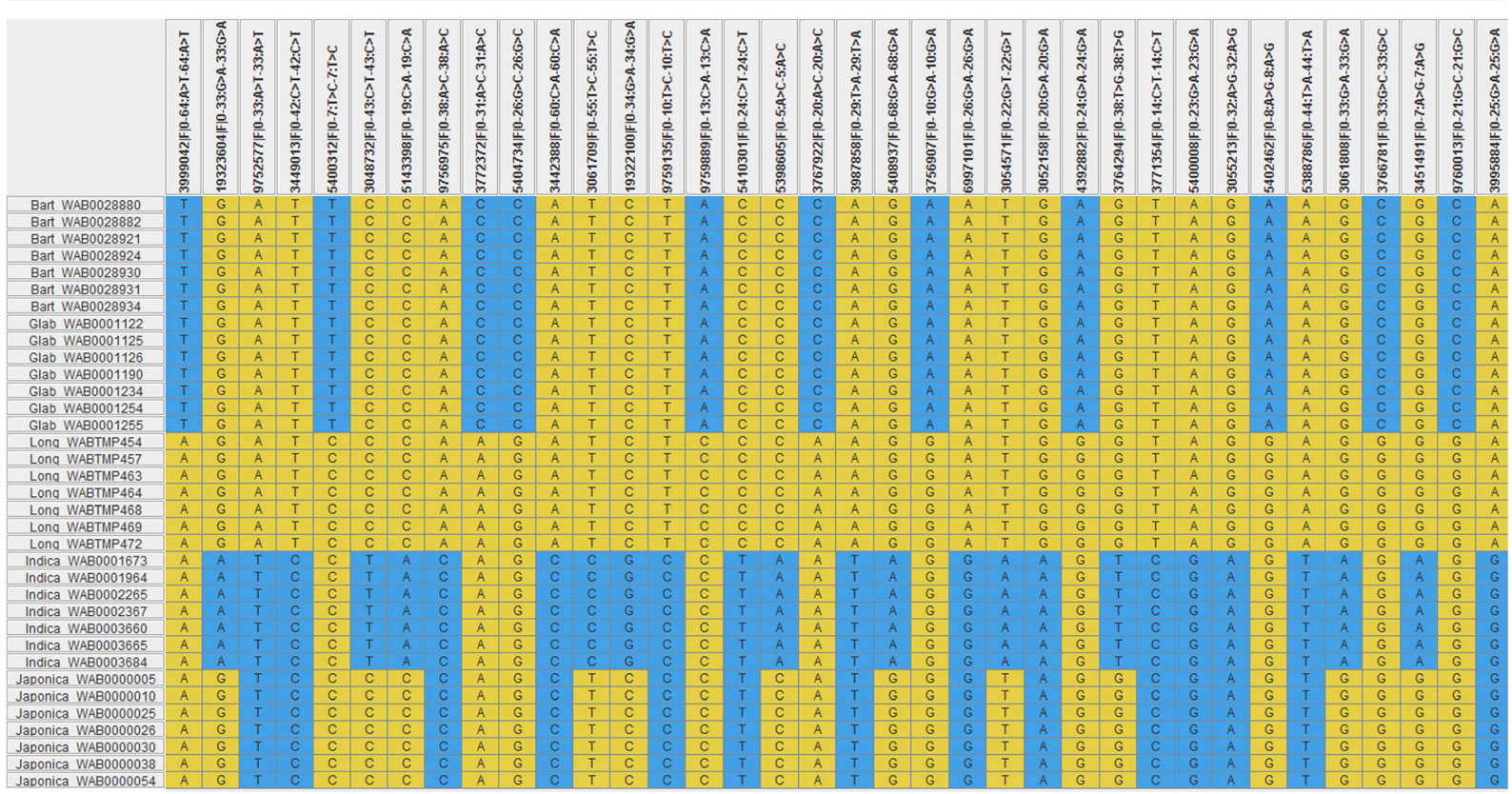

Fig. 4 Haplotype pattern of 36 diagnostic SNP markers recommended for quality control genotyping between the three African species complex and $O$. sativa (14 SNPs), between $O$. longistaminata and $O$. barthii/O. glaberrima (11 SNPs) and between lowland $O$. sativa spp. indica and upland $O$. sativa spp. japonica (11 SNPs). Each species or ecotype is represented by seven randomly selected accessions, which are listed in rows on the left side with prefix (Glab O. glaberrima, Bart O. barthii, Long

samples and 104 randomly selected lines from the germplasm collection. The authors found out that (i) $18 \%$ of the 180 lines were misclassified based on species; (ii) $30 \%$ of the 76 suspected samples were misclassified; (iii) $9 \%$ of the randomly selected samples were misclassified; (iv) several individuals were found to be the product of interspecific hybridization events; and (vi) SNP markers proved to be effective at confirming species identity. Using 53 phenotypic descriptors, approximately $21 \%$ of 3156 yam accessions were found misidentified (not true-to-type individuals) (Girma et al. 2012). Such types of errors can easily be corrected using diagnostic markers and by implementing routine QC genotyping.

To date, a systematic genotyping QC method has been developed and implemented only in maize (Semagn et al. 2012; Ertiro et al. 2015; Chen et al. 2016) to minimize errors associated with genetic purity and genetic identity/authentication. In maize, the focus on QC genotyping was to maintain high genetic purity and consistent genetic identity/authenticity of popular parental lines to minimize use of incorrect parents in
$O$. longistaminata, Indica $O$. sativa spp. indica, japonica $O$. sativa spp. japonica), followed by accession number. The SNP IDs are listed as column headings. Each column has two alleles that are shaded either in orange or blue. See Supplementary Table S1 for details on accessions, Supplementary Table S3 for SNP summary, and Supplementary Table S4 for the genotype data of all diagnostic markers across all 3037 accessions (excluding misclassified accessions)

breeding and genetic studies. In study by the International Maize and Wheat Improvement Center (CIMMYT), for example, researchers compared results from QC genotyping using a smaller set of preselected SNPs with field notes for 280 seed sources of 40 popular parental lines (inbred and doubled haploid lines) and discarded those sources that showed deviation from expectation in terms of purity and identity (Ertiro et al. 2015). In all cases, the decisions made using the preselected SNP markers were as accurate as the field notes recorded on various phenotypic traits. The QC genotyping based on markers was cheaper and faster than evaluating various seed lots in field plots over a period of 3-4 months. In the present study, we used a similar approach as that of maize and verified the 37 accessions genotyped from the original and regenerated seed lots by growing the two seeds lots side by side at the AfricaRice experimental field in Cotonou, Benin (Ndjiondjop et al. 2017). By comparing phenotypic traits recorded on each seed lot with the SNP data, we have corrected the misclassified original seed lots and recommended discarding the regenerated seed lots. 
SNP genotype data can be obtained using one of the numerous uniplex, multiplex, and genotyping by sequencing methods (Semagn et al. 2015). For QC genotyping, a smaller subset of SNPs need to be selected for low-cost uniplex SNP quality control analysis based on ease of scoring with unambiguous separation of homozygous and heterozygous genotypes, minor allele frequency, polymorphism information content, and uniform distribution across chromosomes (Semagn et al. 2012). Using these selection criteria, we recommend between two to three SNPs per chromosome to serve as diagnostic markers in each of the three grups of germplasm. These three groups of germplasm refer to those diagnostic SNPs that separated the three African species complex from the Asian rice, those separating $O$. longistaminata from $O$. glaberrima/O. barthii, and those discriminating lowland $O$. sativa spp. indica from upland $O$. sativa spp. japonica. Of the list of diagnostic markers summarized in Supplementary Table S3 and Supplementary Fig. S2, we recommend developing Kompetitive Allele-Specific PCR (KASP) assays (Semagn et al. 2014) for 36 diagnostic SNPs, with 11-14 SNPs per group of germplasm (Table 1). Researchers from CGIAR and national agricultural research systems (NARS) in developing countries have a contractual agreement with KASP genotyping service providers that costs about US\$ 1.5-2.5 and US\$ 4-5 per sample for a set of 10 and 24 SNPs, respectively, which includes both DNA extraction and SNP genotyping (http://excellenceinbreeding.org/module3). The actual cost per sample varies with the total number of samples to be genotyped. To utilize such services, users can choose 24 of the 36 SNPs (Fig. 4 and Supplementary Table S3) that we recommended for routine QC genotyping in rice. In cases where the QC genotyping should be done within O. sativa germplasm, only 8-11 SNPs that discriminate the lowland $O$. sativa spp. indica from the upland $O$. sativa spp. japonica should be used (Supplementary Table S3). The same is true if the purpose is to discriminate the African species complex from Asian rice or the perennial $O$. longistaminata from the annual $O$. barthii and $O$. glaberrima. Our inability to identify any diagnostic marker between $O$. barthii and $O$. glaberrima was not unexpected. $O$. glaberrima is thought to have evolved from its wild ancestor $O$. barthii through selection (Ogbe and Williams 1978; Linares 2002) and there is evidence showing a wide range of intermediate types between them (Ogbe and Williams 1978). Some diagnostic phenotypic characters such as growth habit, spikelets shattering, and hairiness both on awn and spikelets have been identified. However, such phenotypic traits are often affected by genotype-byenvironment interaction and are not conclusive across diverse accessions. We also did not find any diagnostic SNPs between NERICA and $O$. sativa spp. indica and between NERICA and $O$. sativa spp. japonica. NERICAs were derived from interspecific crosses between $O$. sativa and $O$. glaberrima and selected for adaptation to upland, the rainfed lowland, and the irrigated lowland ecologies across West and Central Africa (Maclean et al. 2002). NERICAs are predominantly $O$. sativa (indica or japonica) background, which is evident from the low proportion of O. glaberrima genome estimated using microsatellite markers (Ndjiondjop et al. 2008) and the moderate genetic differentiation observed between lowland NERICA and lowland $O$. sativa spp. indica $(11.5 \%)$ and between upland NERICA and upland $O$. sativa spp. japonica (6.6\%) using SNP markers (Ndjiondjop et al. 2018). Results from this study would be highly relevant for rice breeders, gene bank managers, and seed system specialists.

\section{Conclusions}

Our results demonstrate the usefulness of genomic tools not only to evaluate the genetic variation and population structure of germplasm conserved in gene banks, but also to detect and correct human errors that could occur at some stage during germplasm collections and routine gene bank operations. Uniplex or multiplex SNP assays can be developed from the sequence information of either all diagnostic SNPs or a subset of preselected species and subspecies (ecotype) diagnostic SNPs that we recommended for routine QC genotyping of at least rice germplasm that are high in demand for distribution to users. About 24 diagnostic SNPs should be sufficient for routine QC genotyping, which includes a set of 8 diagnostic SNPs to discriminate the three African species complex from the $O$. sativa, a second set of 8 SNPs to separate $O$. longistaminata from $O$. glaberrima/O. barthii, and a third set of 8 SNPs to discriminate lowland $O$. sativa spp. indica from upland $O$. sativa spp. japonica. To the best of our knowledge, this is the first extensive study that identified species- and ecotype-specific diagnostic SNPs across multiple Oryza species for low-cost and highthroughput QC genotyping. Using such diagnostic SNP markers, gene bank researchers can determine the identity of their germplasm collections and track misidentification, mislabeling, physical contamination, and loss of genetic integrity. 
Acknowledgements The authors would like to thank Elisée Dannon, Marie Gouette, and Blandine Fatondji for assisting on samples preparation and DNA extraction.

Funding information The present study was financially supported by the Federal Ministry for Economic Cooperation and Development, Germany.

Open Access This article is distributed under the terms of the Creative Commons Attribution 4.0 International License (http:// creativecommons.org/licenses/by/4.0/), which permits unrestricted use, distribution, and reproduction in any medium, provided you give appropriate credit to the original author(s) and the source, provide a link to the Creative Commons license, and indicate if changes were made.

\section{References}

Balasaravanan T, Chezhian P, Kamalakannan R, Yasodha R, Varghese M, Gurumurthi K, Ghosh M (2006) Identification of species-diagnostic ISSR markers for six Eucalyptus species. Silvae Genet 55:119-122

Baloch FS, Alsaleh A, Shahid MQ, Çiftçi V, Sáenz De Miera LE, Aasim M, et al. (2017) A whole genome DArTseq and SNP analysis for genetic diversity assessment in durum wheat from central fertile crescent. PLoS One 12:e0167821

Bradbury PJ, Zhang Z, Kroon DE, Casstevens TM, Ramdoss Y, Buckler ES (2007) TASSEL: software for association mapping of complex traits in diverse samples. Bioinformatics 23: 2633-2635

Buso GSC, Rangel PHN, Ferreira ME (2001) Analysis of random and specific sequences of nuclear and cytoplasmic DNA in diploid and tetraploid American wild rice species (Oryza spp.). Genome 44:476-494

Chen C, He W, Nassirou TY, Nsabiyumva A, Dong X, Adedze YMN, Jin D (2017) Molecular characterization and genetic diversity of different genotypes of Oryza sativa and Oryza glaberrima. Electron J Biotechnol 30:48-57

Chen J, Zavala C, Ortega N, Petroli C, Franco J, Burgueño J, Costich DE, Hearne SJ (2016) The development of quality control genotyping approaches: a case study using elite maize lines. PLoS One 11:e0157236

Chin JH, Kim J-H, Jiang W, S-h C, Mo W, Lz H et al (2007) Identification of subspecies-specific STS markers and their association with segregation distortion in rice (Oryza Sativa L.). J Crop Sci Biotech 10:175-184

Cubry P, Tranchant-Dubreuil C, Thuillet A-C, Monat C, Ndjiondjop M-N, Labadie K, et al. (2018) The rise and fall of African rice cultivation revealed by analysis of 246 new genomes. Curr Biol 28:(in press)

Cullingham CI, Cooke JEK, Dang S, Coltman DW (2013) A species-diagnostic SNP panel for discriminating lodgepole pine, jack pine, and their interspecific hybrids. Tree Genetics and Genomes 9:1119-1127

Curk F, Ancillo G, Ollitrault F, Perrier X, Jacquemoud-Collet J-P, Garcia-Lor A, Navarro L, Ollitrault P (2015) Nuclear species-diagnostic SNP markers mined from 454 amplicon sequencing reveal admixture genomic structure of modern citrus varieties. PLoS One 10:e0125628

Elshire RJ, Glaubitz JC, Sun Q, Poland JA, Kawamoto K, Buckler ES, Mitchell SE (2011) A robust, simple genotyping-bysequencing (GBS) approach for high diversity species. PLoS One 6:e19379

Ertiro BT, Ogugo V, Worku M, Das B, Olsen M, Labuschagne M, Semagn K (2015) Comparison of Kompetitive Allele Specific PCR (KASP) and genotyping by sequencing (GBS) for quality control analysis in maize. BMC Genomics 16:908

Frey JE, Guillén L, Frey B, Samietz J, Rull J, Aluja M (2013) Developing diagnostic SNP panels for the identification of true fruit flies (Diptera: Tephritidae) within the limits of COIbased species delimitation. BMC Evol Biol 13:106

Ge S, Sang T, Lu BR, Hong DY (2001) Phylogeny of the genus Oryza as revealed by molecular approaches. In: Khush GS, Brar DS, Hardy B (eds) Rice genetics IV Proceedings of the fourth international rice genetics symposium. International Rice Research Institute (IRRI), Los Banos, Philippines

Girma G, Korie S, Dumet D, Franco J (2012) Improvement of accession distinctiveness as an added value to the global worth of the yam (Dioscorea spp.) genebank. International Journal of Conservation Science 3:199-206

Jones MP, Dingkuhn M, GK A/s, Semon M (1997) Interspecific Oryza sativa L. x O. glaberrima Steud. progenies in upland rice improvement. Euphytica 94:237-246

Joshi SP, Gupta VS, Aggarwal RK, Ranjekar PK, Brar DS (2000) Genetic diversity and phylogenetic relationship as revealed by inter simple sequence repeat (ISSR) polymorphism in the genus Oryza. Theor Appl Genet 100:1311-1320

Kshirsagar SS, Samal KC, Rabha M, Bastia DN, Rout GR (2014) Identification of variety diagnostic molecular marker of high yielding rice varieties. Proc Natl Acad Sci, India, Sect B Biol Sci 84:389-396

Kumar S, Stecher G, Tamura K (2016) MEGA7: molecular evolutionary genetics analysis version 7.0 for bigger datasets. Mol Biol Evol 33:1870-1874

Linares OF (2002) African rice (Oryza glaberrima): history and future potential. Proc Natl Acad Sci 99:16360-16365

Maclean JL, Dawe DC, Hardy B, Hettel GP (2002) Rice almanac. CABI Publishing, Los Banos, Philippines

Mason AS, Zhang J, Tollenaere R, Vasquez Teuber P, DaltonMorgan J, Hu L, Yan G, Edwards D, Redden R, Batley J (2015) High-throughput genotyping for species identification and diversity assessment in germplasm collections. Mol Ecol Resour 15:1091-1101

McCouch SR, McNally KL, Wang W, Hamilton RS (2012) Genomics of gene banks: a case study in rice. Am J Bot 99: 407-423

Melville J, Haines ML, Boysen K, Hodkinson L, Kilian A, Smith Date KL, Potvin DA, Parris KM (2017) Identifying hybridization and admixture using SNPs: application of the DArTseq platform in phylogeographic research on vertebrates. Royal Soc Open Sci 4

Milne I, Shaw P, Stephen G, Bayer M, Cardle L, Thomas WTB, Flavell AJ, Marshall D (2010) Flapjack-graphical genotype visualization. Bioinformatics 26:3133-3134

Ndjiondjop M-N, Semagn K, Gouda AC, Kpeki SB, Dro Tia D, Sow M, et al. (2017) Genetic variation and population 
structure of Oryza glaberrima and development of a minicore collection using DArTseq. Front Plant Sci 8:1748

Ndjiondjop MN, Semagn K, Sie M, Cissoko M, Fatondji B, Jones M (2008) Molecular profiling of interspecific lowland rice populations derived from IR64 (Oryza sativa) and Tog5681 (Oryza glaberrima). Afr J Biotechnol 7:4219-4229

Ndjiondjop MN, Semagn K, Sow M, Manneh B, Gouda AC, Kpeki SB, Pegalepo E, Wambugu P, Sié M, Warburton ML (2018) Assessment of genetic variation and population structure of diverse rice genotypes adapted to lowland and upland ecologies in Africa using SNPs. Front Plant Sci 9:446

Ogbe FMD, Williams JT (1978) Evolution in indigenous west African rice. Econ Bot 32:59-64

Orjuela J, Sabot F, Chéron S, Vigouroux Y, Adam H, Chrestin H, Sanni K, Lorieux M, Ghesquière A (2014) An extensive analysis of the African rice genetic diversity through a global genotyping. Theor Appl Genet 127:2211-2223

Rafalski A (2002) Applications of single nucleotide polymorphisms in crop genetics. Curr Opin Plant Biol 5:94-100

Sansaloni C, Petroli C, Jaccoud D, Carling J, Detering F, Grattapaglia D, Kilian A (2011) Diversity Arrays Technology (DArT) and next-generation sequencing combined: genomewide, high throughput, highly informative genotyping for molecular breeding of Eucalyptus. BMC Proc 5:P54

Schlotterer C (2004) The evolution of molecular markers - just a matter of fashion? Nat Rev Genet 5:63-69

Semagn K, Babu R, Hearne S, Olsen M (2014) Single nucleotide polymorphism genotyping using Kompetitive Allele Specific
PCR (KASP): overview of the technology and its application in crop improvement. Mol Breed 33:1-14

Semagn K, Beyene Y, Makumbi D, Mugo S, Prasanna BM, Magorokosho C, Atlin G (2012) Quality control genotyping for assessment of genetic identity and purity in diverse tropical maize inbred lines. Theor Appl Genet 125:1487-1501

Semagn K, Beyene Y, Raman B, Sudha N, Gowda M, Biswanath $\mathrm{D}$ et al (2015) Quantitative trait loci mapping and molecular breeding for developing stress resilient maize for subSaharan Africa. Crop Sci 55:1449-1459

Semon M, Nielsen R, Jones MP, McCouch SR (2005) The population structure of African cultivated rice Oryza glaberrima (Steud.): evidence for elevated levels of linkage disequilibrium caused by admixture with $O$. sativa and ecological adaptation. Genetics 169:1639-1647

Wambugu PW, Furtado A, Le Waters D, Nyamongo DO, Henry RJ (2013) Conservation and utilization of African Oryza genetic resources. Rice 6:1-13

Yan J, Yang X, Shah T, Sanchez-Villeda H, Li J, Warburton M et al (2010) High-throughput SNP genotyping with the GoldenGate assay in maize. Mol Breed 25:441-451

Zhao X, Yang L, Zheng Y, Xu Z, Wu W (2009) Subspeciesspecific intron length polymorphism markers reveal clear genetic differentiation in common wild rice (Oryza rufipogon L.) in relation to the domestication of cultivated rice (O. sativa L.). J Genet Genomics 36:435-442 